\title{
Efecto de un programa de reacondicionamiento físico sobre la movilidad y fuerza muscular en personas adultas mayores con enfermedad pulmonar crónica
}

\author{
Heyden López, Franklin; Muñoz Rojas, Derby \\ Efecto de un programa de reacondicionamiento físico sobre la movilidad y fuerza muscular en personas adultas \\ mayores con enfermedad pulmonar crónica \\ MHSalud, vol. 18, núm. 1, 2021 \\ Universidad Nacional, Costa Rica \\ Disponible en: http://www.redalyc.org/articulo.oa?id=237064193007 \\ DOI: https://doi.org/10.15359/mhs.18-1.7
}

Esta obra está bajo una Licencia Creative Commons Atribución-NoComercial-SinDerivar 3.0 Internacional. 


\title{
Efecto de un programa de reacondicionamiento físico sobre la movilidad y fuerza muscular en personas adultas mayores con enfermedad pulmonar crónica
}

\author{
Effects of a Physical Conditioning Program on the Mobility and Muscle Strength of Older Adults with Chronic \\ Lung Disease \\ Efeito de um programa de recondicionamento físico na mobilidade e na força muscular em pessoas idosas com \\ doença pulmonar crônica
}

Franklin Heyden López

Caja Costarricense de Seguro Social, Costa Rica

fheyden@ccss.sa.cr

DOI: https://doi.org/10.15359/mhs.18-1.7 Redalyc: http://www.redalyc.org/articulo.oa?

(D) http://orcid.org/0000-0002-1204-3599

Derby Muñoz Rojas

Universidad de Costa Rica, Costa Rica

derby.munoz@ucr.ac.cr

(iD http://orcid.org/0000-0003-2143-4716 $\mathrm{id}=237064193007$

Recepción: 08 Febrero 2020 Aprobación: 23 Septiembre 2020

\section{ReSUMEN:}

Los problemas respiratorios son una de las principales causas de morbilidad en las personas adultas mayores. Las enfermedades pulmonares crónicas afectan la capacidad ventilatoria y limitan la habilidad física de los pacientes, lo que tiene consecuencias negativas para la calidad de vida. Por esta razón, los programas de rehabilitación pulmonar intentan reacondicionar la capacidad física y así mejorar el bienestar de esa población. El estudio tiene por objetivo determinar el efecto de un programa de reacondicionamiento físico en personas adultas mayores con enfermedad pulmonar crónica sobre la movilidad y la fuerza muscular, para lo cual se realizó un análisis retrospectivo del efecto de un plan de ejercicio físico en la movilidad y fuerza muscular de 53 personas adultas con enfermedad pulmonar crónica. Se recolectaron datos en el Hospital Nacional de Geriatría y Gerontología, de personas que participaron en dicho plan entre enero de 2012 y diciembre de 2017. Este tiene una duración de 16 semanas; se compone de evaluaciones y trabajo específico aeróbico, contra resistencia, funcional, más fisioterapia respiratoria. La información fue examinada utilizando pruebas pareadas de t-student, lo que permitió comprobar que hubo mejoras estadísticamente significativas en la fuerza muscular $(\mathrm{p}<0.001)$ y la movilidad $(\mathrm{p}<0.001)$ de los sujetos, luego de participar en el programa de reacondicionamiento. Se concluye que la prescripción efectiva de ejercicio para las personas adultas mayores con enfermedad pulmonar crónica incluye la planificación de trabajo aeróbico, contrarresistencia, actividad funcional, fisioterapia respiratoria, lo que impactaría positivamente su movilidad y fuerza muscular.

Palabras Clave: adulto mayor, ejercicio de rehabilitación, enfermedad pulmonar, fuerza muscular.

\section{Abstract:}

Respiratory problems are one of the major causes of morbidity in older adults. Chronic lung diseases affect the ventilatory capacity, limiting patients' physical ability, which negatively affects their quality of life. Thus, pulmonary rehabilitation programs try to recondition patients' physical capacity; as a result, their well-being improves. This study was conducted to determine the effect of a physical reconditioning program in older people with chronic lung disease; for this, a retrospective analysis of the effect of a physical exercise plan was conducted. The variables included in the study were mobility and muscle strength. Data were collected in the National Hospital of Geriatrics and Gerontology from 53 participants who attended the program between January 2012 and December 2017. This program lasts 16 weeks and comprises assessments and specific work sessions on aerobic resistance, strength, functional exercise, and respiratory physiotherapy. Results from paired $t$-student tests showed that there was a statistically significant improvement in muscular strength $(\mathrm{p}<0.001)$ and mobility $(\mathrm{p}<0.001)$ of the subjects after participating in the physical reconditioning program. It is concluded that the effective prescription of exercise for the elderly with chronic lung disease should include work on aerobic resistance, strength, functional exercise, and respiratory physiotherapy, which would positively affect agility, balance, strength, and muscular endurance.

KEYWORDS: elderly, exercise therapy, lung disease, muscle strength. 
MHSALUD, ISSN: 1659-097X, 18(1), ENERO-Junio, 2021, PP 1-11

Heyden López y Muñoz RoJas

\section{Resumo:}

Problemas respiratórios são uma das principais causas de morbidade em pessoas idosas. As doenças pulmonares crônicas afetam a capacidade ventilatória, limitando a capacidade física 2 pacientes, o que traz consequências negativas para a qualidade de vida. Por isso, os programas de reabilitação pulmonar buscam recondicionar a capacidade física e, assim, melhorar o bem-estar dessa população. O objetivo desta pesquisa é determinar o efeito de um programa de recondicionamento físico em pessoas idosas com doença pulmonar crônica na mobilidade e na força muscular. Foi realizada uma análise retrospectiva do efeito de um programa de exercícios físicos na mobilidade e força muscular em 53 adultos com doença pulmonar crônica. Os dados foram coletados no Hospital Nacional de Geriatria y Gerontologia, de pessoas que participaram do programa entre janeiro de 2012 e dezembro de 2017. O programa tem duração de 16 semanas, e consiste em avaliações, além de trabalhos específicos de aeróbica, de resistência, funcionais e fisioterapia respiratória. Os dados foram analisados por meio de testes $t$-student pareados, o que permitiu verificar que houve melhora estatisticamente significativa na força muscular $(\mathrm{p}<0,001)$ e mobilidade $(\mathrm{p}<0,001) 2$ sujeitos após a participação no programa de recondicionamento. Conclui-se que a prescrição efetiva de exercícios para pessoas idosas com doença pulmonar crônica inclui a programação do trabalho aeróbico, contra resistência, trabalho funcional, fisioterapia respiratória, o que impactaria positivamente na mobilidade e na força muscular.

PAlaVRas-CHAVE: pessoa idosa, exercício de reabilitação, doença pulmonar, força muscular.

\section{INTRODUCCIÓN}

La Caja Costarricense de Seguro Social reportó que las enfermedades respiratorias ocuparon, en el nivel nacional, el sexto lugar de egresos hospitalarios, según un diagnóstico en el 2016, con 23864 casos que correspondieron al $67 \%$ de los reportes (CCSS, 2016). Similarmente, en el Hospital Nacional de Geriatría y Gerontología-Caja Costarricense de Seguro Social (HNGG-CCSS), para el mismo año se reportó que el $12.3 \%(\mathrm{n}=317)$ de las causas de egreso fueron asociadas a problemas respiratorios (HNGG, 2016). En relación con las causas de mortalidad, el mismo hospital reportó que, para el 2016, del total de defunciones un $18.5 \%(n=69)$ correspondió a enfermedades respiratorias. Lo anterior ciertamente refleja el patrón descrito en el Informe Estado de la Nación del 2013, el cual menciona que en el 2011 las enfermedades del sistema respiratorio ocuparon el cuarto lugar de razón de muerte, según grupo de causas (Rayo, 2013).

La literatura ha identificado diversas consecuencias asociadas a las enfermedades pulmonares, incluyendo una disminución en la calidad de vida, la capacidad aeróbica y la masa muscular (Satta et al., 1997; Sivori et al., 2008). Los adultos mayores con problema pulmonar presentan disnea, la cual los lleva a limitar sus actividades físicas, puesto que causa debilidad muscular y deteriora sus funciones; así, la sensación de dificultad respiratoria puede incluso incrementarse (proceso conocido como la espiral de la disnea (AACVPR, 2011). Además, en la enfermedad obstructiva, los músculos inspiratorios trabajan en condiciones mecánicas desfavorables, a mayor velocidad y contra más resistencia elástica, generando disnea y fatiga de estos. En tanto, en la enfermedad restrictiva, se produce una disminución en la distensibilidad y el volumen pulmonares, ocasionando, también, disnea y fatiga (Moore et al., 2016).

Diversos organismos internacionales han recomendado la implementación de programas de rehabilitación pulmonar (RP), la cual, de acuerdo con la Sociedad Americana de Tórax y la Sociedad Respiratoria Europea, se define como una intervención basada en la evaluación del paciente y con tratamientos que incluyen, pero no se limitan a, entrenamiento, educación y cambios de comportamiento, diseñado para mejorar la condición física y psicológica de las personas con enfermedades respiratorias crónicas y promover cambios hacia un estilo de vida saludable a largo plazo (Spruit et al., 2013).

La literatura ha identificado que los programas de RP enfocados en el reacondicionamiento físico poseen múltiples beneficios para la persona. En el impacto positivo de estos programas, se pueden citar: mejora de la capacidad de ejercicio y de la calidad de vida relacionada con la salud; menor percepción de disnea; disminución de ingresos hospitalarios y días de internamientos; decrecimiento de la ansiedad y la depresión asociada a la Enfermedad Pulmonar Crónica (EPC); todos estos beneficios son reportados con un nivel de evidencia A (Sivori et al., 2008; Ries et al., 2007). El entrenamiento físico es el componente principal de la 
rehabilitación pulmonar (Marco et al., 2016), permite el reacondicionamiento físico. La fragilidad del adulto mayor se caracteriza por del deterioro de sus reservas fisiológicas, manifestado en 5 elementos: pérdida de peso, sarcopenia, fatiga, marcha inestable o lenta y actividad física mínima. Tales condiciones se suman al problema pulmonar que afecta a la población adulta mayor (Morales et al., 2017).

Con respecto a la estructuración de los programas de reacondicionamiento físico, un aspecto fundamental es la evaluación, la cual debe aplicarse al inicio y al final e incluir, como mínimo, valoración del grado de disnea, de la función respiratoria, de la capacidad aeróbica y de la calidad de vida relacionada con salud (Sivori et al., 2008; Marco et al., 2016 ). De la misma manera, según el nivel con el que se evidencie la eficiencia de la rehabilitación respiratoria, de acuerdo con la Sociedad Americana de Tórax y la Sociedad de Rehabilitación Cardiorrespiratoria, los programas deben incluir: entrenamiento de músculos periféricos (piernas y brazos, con grado de recomendación 1A), trabajos de pesas con los miembros superiores, tareas de resistencia aeróbica con miembros inferiores y ejercicios de músculos respiratorios - con gado de recomendación 1B - (Marco et al., 2016), sin dejar de lado la fisioterapia y la reeducación respiratorias (Miranda et al., 2011; Marco et al., 2016).

En el HNGG-CCSS, se ofrece el Programa de Reacondicionamiento Físico para Adulto Mayor con Enfermedad Pulmonar Crónica (PRFAMEPC), que incluye alteraciones obstructivas y restrictivas. Forma parte de la fase 2 de un Programa de RP implementado en el hospital desde el 2012.

A pesar de que en Costa Rica se está empezando a incursionar en la RP, existe limitada evidencia sobre el impacto de los programas mencionados en la persona adulta mayor con problemas pulmonares. Por ello, el propósito del trabajo fue establecer el efecto de un programa de reacondicionamiento físico para adulto mayor con problema pulmonar crónico sobre la movilidad y la fuerza muscular, con las siguientes hipótesis: $\mathrm{H} 1$, la movilidad mejora luego de participar en el PRFAMEPC y H2, la fuerza muscular mejora luego de participar en el PRFAMEPC.

\section{Metodología}

\section{Participantes}

Para el desarrollo de este trabajo, se utilizó un diseño descriptivo, retrospectivo, con análisis de registros médicos de las personas que participaron en el PRFAMEPC del HNGG-CCSS. El siguiente corresponde al diseño observado en el PRFAMEPC del HNGG-CCSS, en el cual se realiza una evaluación inicial, aplicación del programa y se concluye con una evaluación final.

$$
\mathrm{G}=\mathrm{O} 1 \mathrm{XO} 2 \mathrm{G}=\text { Grupo; } \mathrm{O}=\text { evaluación; } \mathrm{X}=\text { tratamiento }
$$

Para ingresar al programa, la persona debe ser referida por el médico especialista. De quienes participaron del plan, se seleccionó una muestra de 53 usuarios, mayores de 65 años, sin restricción de género o etnia, los cuales formaron parte del programa entre enero del 2012 y diciembre del 2017. Se identificaron como obstructivos (valores espirométricos con VEF1/CVF menor del $70 \%$ del predicho y CVF menor del 80 $\%$ del predicho) o restrictivos (valores espirométricos con VEF1/CVF mayor o igual al $70 \%$ del predicho y CVF menor del $80 \%$ del predicho). Todos los procedimientos de este trabajo fueron aprobados por el Comité Ético Científico del HNGG-CCSS, protocolo HNGG-CEC 04-2018, y se inscribieron en el Consejo Nacional de Investigación en Salud. 


\section{Instrumentos}

Senior Fitness Test: Es una batería de pruebas que evalúa la capacidad funcional del adulto mayor, entendida como la condición física para realizar actividades de la vida cotidiana. Entre sus variables incluye fuerza muscular (miembros superiores e inferiores), resistencia aeróbica, flexibilidad (miembros superiores e inferiores) y movilidad (Rikli y Jones, 2001).

Para efecto del estudio, se utilizó lo expuesto a continuación:

- La prueba de fuerza de miembros inferiores, que consiste en sentarse y levantarse de una silla la mayor cantidad de veces posibles. Es medida por la cantidad de repeticiones y el percentil de movimientos dinámicos en miembros inferiores logrados en 30 segundos. Tiene una correlación, con un goniómetro, de 0.81 para mujeres y 0.76 para hombres (Jones et al., 1998). Se considera que el rango de percentil normal está entre 25 y 75 , mientras que el bajo es $<25$ y el alto $>75$ (Rikli y Jones, 2001).

- La prueba de fuerza de miembros superiores, que se trata de realizar la mayor cantidad de flexiones y extensiones del codo (i. e., utilizando 5 libras para mujeres y 8 libras para hombres). Es medida por la cantidad de repeticiones y el percentil de movimientos dinámicos en miembros superiores logrados en 30 segundos. Tiene una validez con un $r=0,82$ de correlación, con Cybex Machine Arm Curl Performance (Osness et al., 1996). El rango de percentil normal se encuentra entre 25 y 75 , el bajo es $<25$ y el alto $>75$ (Rikli y Jones, 2001).

- La prueba de valoración de la movilidad, que envuelve velocidad, coordinación y balance postural. Es medida por el número de segundos requeridos para incorporarse desde una posición sedente, caminar 2.44 metros, girar y regresar hasta sentarse. Estas acciones se valoran en segundos y se clasifican en percentil. A pesar de que no hay una medición de oro contra la cual comparar, esta prueba tiene una relación significativa, con la Berg Balance Scale, de 0.81 y, con el índice de Barthel, de 0.78 (Podsiadlo y Richardson, 1991). El rango de percentil normal está entre 25 y 75 , mientras que el bajo se encuentra en $<25$ y el alto es $>75$ (Rikli y Jones, 2001).

\section{Procedimiento}

Se tomaron los resultados pre y post del Senior Fitness Test, sobre los componentes de fuerza muscular de miembros superiores e inferiores, así como la valoración de la movilidad de las personas adultas mayores con enfermedad pulmonar crónica que participaron del PRFAMEPC. Los datos fueron extraídos de los registros médicos, de acuerdo con la selección de las variables de interés, sin incluir información que permita identificar al participante.

El programa se desarrolló en un total de 16 semanas, distribuidas de la siguiente manera: 2 de evaluación inicial, 2 de evaluación final y 12 ( 24 sesiones) de trabajo específico, que incluyó labor aeróbica de 12 semanas, tareas contrarresistencia (pesas) de 10 semanas, ejercicio funcional de 10 semanas y fisioterapia respiratoria que incorporó la reeducación ventilatoria de 12 semanas. A continuación, en las tablas 1 y 2 , se describe la estructura del programa. 
MHSALUD, ISSN: 1659-097X, 18(1), ENERO-Junio, 2021, PP 1-11 Heyden López y MuÑoz RoJas

TABLA 1

Programa de Reacondicionamiento Físico para Adulto Mayor con Enfermedad Pulmonar Crónica

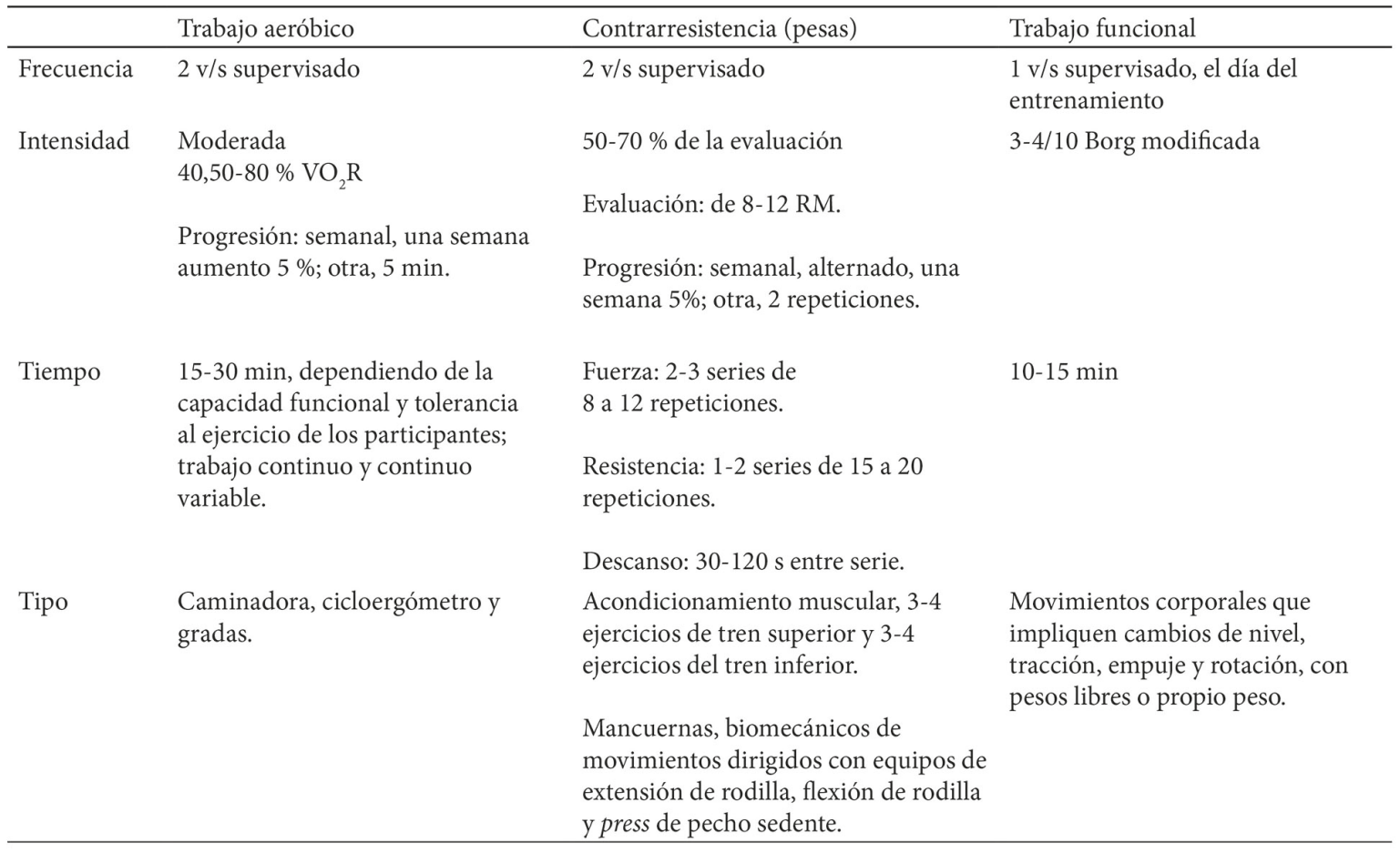

Min = minutos $\mathrm{RM}=$ repeticiones máximas; $\mathrm{s}=$ segundo; $\mathrm{VO} 2 \mathrm{R}=$ consumo de oxígeno reserva; $\mathrm{v} / \mathrm{s}=\mathrm{veces}$ por $\mathrm{semana}$.

TABLA 2

Distribución del componente de la fisioterapia respiratoria en el PRFAMEPC

\begin{tabular}{|c|c|c|c|c|c|}
\hline & $\begin{array}{l}\text { Reeducación } \\
\text { ventilatoria }\end{array}$ & Higiene bronquial & Expansión pulmonar & & $\begin{array}{l}\text { Entrenamiento de la } \\
\text { musculatura ventilatoria }\end{array}$ \\
\hline Frecuencia & $5-6 \mathrm{v} / \mathrm{s}$ & $\begin{array}{l}\text { En presencia de } \\
\text { secreciones. }\end{array}$ & $\begin{array}{l}\text { Semana } 1-2,2 \mathrm{v} / \mathrm{d} \text {, } \\
5-6 \mathrm{v} / \mathrm{s} \text {. } \\
\text { Semana } 3-4,2 \mathrm{v} / \mathrm{d} \text {, } \\
3 \mathrm{v} / \mathrm{s} \text {. }\end{array}$ & $\begin{array}{l}\text { Semana 3-12, } 2 \\
\text { v/d, } 3 \text { v/s. (martes, } \\
\text { jueves y sábado). }\end{array}$ & $\begin{array}{l}\text { Semana 3-12, } 2 \text { v/d, } 3 \\
\text { v/s (lunes, miércoles y } \\
\text { viernes). }\end{array}$ \\
\hline Intensidad & $\begin{array}{l}1-2 / 10 \text { Borg } \\
\text { modificado }\end{array}$ & $\begin{array}{l}1-3 \text { series, } \\
10-15 \text { repeticiones }\end{array}$ & $\begin{array}{l}\text { 1-3 series, } \\
\text { 10-15 repeticiones } \\
\text { Descanso: } 30-120 \mathrm{~s} \\
\text { entre series. } \\
\text { Progresión: } \\
\text { semanal, alternado, } \\
\text { una semana } \\
\text { alrededor de } \\
125 \text { ml; otra, } 2 \\
\text { repeticiones. }\end{array}$ & $\begin{array}{l}\text { 6-12 cmH2O, } \\
\text { 4-6 series, } \\
\text { 10-20 repeticiones } \\
\text { Descanso: } 30-120 \mathrm{~s} \\
\text { entre series. } \\
\text { Progresión: } \\
\text { semanal, alternado, } \\
\text { una semana } 5 \% \\
\text { intensidad; otra, } 2 \\
\text { repeticiones. }\end{array}$ & $\begin{array}{l}\text { 40-60 \% de la PImáx, } \\
\text { 4-6 series, } \\
\text { 10-20 repeticiones } \\
\text { Descanso: } 30-120 \mathrm{~s} \\
\text { entre series. } \\
\text { Progresión: semanal, } \\
\text { alternado, una semana } \\
5 \% \text { intensidad; otra, } 2 \\
\text { repeticiones. }\end{array}$ \\
\hline Tiempo & $5-10 \mathrm{~min}$ & & & & \\
\hline Tipo & $\begin{array}{l}\text { Respiración } \\
\text { diafragmática, } \\
\text { ejercicios para } \\
\text { musculatura } \\
\text { inspiratoria }\end{array}$ & $\begin{array}{l}\text { Dispositivo } \\
\text { de oscilación } \\
\text { vibratoria }\end{array}$ & $\begin{array}{l}\text { Inspirómetro } \\
\text { incentivo de } \\
\text { volumen }\end{array}$ & $\begin{array}{l}\text { DPEP de umbral } \\
\text { no oscilante }\end{array}$ & IMT umbral \\
\hline
\end{tabular}




\section{Análisis estadísticos}

Todos los datos fueron exportados a SPSS.24.0 $0^{\circ}$ Posteriormente se realizaron los análisis descriptivos, utilizando medidas de tendencia central que incluían estimación de promedio y desviación estándar. Además, se ejecutaron análisis paramétricos con el fin de identificar el efecto del programa, incluyendo pruebas de $\mathrm{t}$ student. Para el manejo de los datos perdidos, se reconocieron en el sistema; luego se revisaron e imputaron. $\mathrm{Al}$ repasar estos procesos, se compararon la inclusión y exclusión de los datos perdidos y se comprobó su remoción no influyó en el comportamiento de lo observado.

\section{Resultados}

En la tabla 3 se muestran las características de la población.

TABLA 3

Características de la población $N=53$

\begin{tabular}{lccc}
\hline & $\begin{array}{c}\text { Hombres } \\
(n=33)\end{array}$ & $\begin{array}{c}\text { Mujeres } \\
(n=20)\end{array}$ & Total \\
\hline Edad (años) & $77.45 \pm 6.02$ & $77.85 \pm 5.50$ & $77.60 \pm 5.78$ \\
Obstructivo & 26 & 19 & 45 \\
Restrictivo & 7 & 1 & 8 \\
\hline
\end{tabular}

Nota: Datos son presentados como media y desviación estándar.

Fuerza muscular: De acuerdo con la tabla 4, se encontró que el PRFAMEPC tuvo un efecto significativo sobre la fuerza muscular de miembros inferiores y superiores, evaluada por el Senior Fitness Test, entre las mediciones aplicadas en el grupo de adultos mayores con EPC.

TABLA 4

Efecto del programa en la fuerza muscular

\begin{tabular}{cccccccc}
\hline & $n$ & Pre $\pm D E$ & Post $\pm D E$ & $\begin{array}{c}\text { Diferencia } \\
\text { promedio }\end{array}$ & $t$ & $g l$ & $p$ \\
\hline FMMI (repeticiones) & 47 & $8.70 \pm 3.85$ & $11.38 \pm 4.01$ & -2.68 & -6.97 & 46 & $0.000^{* *}$ \\
FMMI (percentil) & 46 & $23.63 \pm 20.97$ & $40.35 \pm 25.55$ & -16.72 & -6.79 & 45 & $0.000^{* *}$ \\
FMMS (repeticiones) & 50 & $10.12 \pm 4.30$ & $13.02 \pm 4.57$ & -2.90 & -8.90 & 49 & $0.000^{* *}$ \\
FMMS (percentil) & 49 & $20.57 \pm 16.67$ & $38.92 \pm 23.40$ & -18.34 & -7.85 & 48 & $0.000^{* *}$ \\
\hline
\end{tabular}

Nota: FMMI = fuerza muscular de miembros inferiores; FMMS = fuerza muscular de miembros superiores. ${ }^{* *} \mathrm{p}=$ valor $<0.001$.

Por lo tanto, se acepta la hipótesis de que la fuerza muscular de una persona adulta mayor con enfermedad pulmonar crónica mejora luego de participar en el PRFAMEPC.

Movilidad: Se encuentran diferencias estadísticamente significativas en la movilidad evaluada por la velocidad, la coordinación y el balance postural del Senior Fitness Test, entre las mediciones aplicadas en el grupo evaluado de adultos mayores con problema pulmonar crónico (tabla 5). 
TABLA 5

Efecto del programa en la movilidad

\begin{tabular}{cccccccc}
\hline & $n$ & Pre $\pm D E$ & Post $\pm D E$ & $\begin{array}{c}\text { Diferencia } \\
\text { promedio }\end{array}$ & $t$ & $g l$ & $p$ \\
\hline VCBP (s) & 47 & $9.00 \pm 2.96$ & $8.39 \pm 4.80$ & 0.61 & 1.02 & 46 & 0.312 \\
VCBP (percentil) & 47 & $20.90 \pm 20.37$ & $32.54 \pm 24.26$ & -11.64 & -5.58 & 46 & $0.000^{* *}$ \\
\hline
\end{tabular}

Nota: VCBP = Velocidad, coordinación y balance postural. ${ }^{* *} \mathrm{p}=$ valor $<0.001$.

Por lo tanto, se acepta la hipótesis de que la movilidad de una persona adulta mayor con enfermedad pulmonar crónica mejora luego de participar en el PRFAMEPC.

\section{Discusión}

Este estudio tuvo como objetivo identificar el efecto de un programa de reacondicionamiento físico en personas adultas mayores con EPC, por lo que se analizaron sus consecuencias sobre la fuerza muscular y la movilidad. Se comprobaron las hipótesis, al confirmar que ambas características de las personas adultas mayores con EPC mejoraron luego de participar en el PRFAMEPC.

En la programación del ejercicio, se incluyó un componente específico de contrarresistencia, el cual fue complementario al aeróbico, ya que este tipo de trabajo requiere una menor demanda ventilatoria y provoca una mejora tanto en la fuerza como en la resistencia muscular (AACVPR, 2011; De Brandt et al., 2018; Güell et al., 2014). Con respecto a esta complementariedad en la prescripción del ejercicio, se ha encontrado en la literatura que el efecto en la fuerza de las tareas combinadas aeróbicas y contrarresistencia es mayor que cuando se programa únicamente un tipo de entrenamiento, debido a que se inducen cambios morfológicos (e. g., se contrarresta la atrofia del músculo esquelético) y funcionales (De Brandt et al., 2018). Similarmente, se ha encontrado, de modo consistente, que el ejercicio en la rehabilitación pulmonar aumenta la función muscular y retrasa la fatiga, lo que deriva en una mayor tolerancia al ejercitación (McCarthy, 2015).

Los resultados coinciden con estudios previos, en los cuales se ha encontrado que el entrenamiento de los miembros superiores incrementa la resistencia de los brazos, modula la hiperinflación dinámica y reduce los síntomas tanto de disnea como de fatiga muscular, mientras que el trabajo contrarresistencia de los miembros inferiores mejora la tolerancia al ejercicio (Janaudis-Ferreira, 2011; Marco et al., 2016). Además, la fuerza en los miembros inferiores se acrecienta, debido al entrenamiento de pesas y trabajo aeróbico, lo cual concuerda con Marco et al. (2016).

La medida del percentil de la fuerza muscular de miembros superiores e inferiores, así como la movilidad (i. e., agilidad y equilibrio) pasaron de valores por debajo del promedio a otros dentro de lo normal para la edad, según lo establecido por Rikli y Jones (2001). Se ratifica el incremento de la fuerza y la movilidad observadas en los participantes. Complementariamente, esta mejora en la fuerza de los miembros inferiores podría generar mayor distancia en la caminata de 6 minutos y, por ende, en la movilidad de la persona. El hecho es consistente con las recomendaciones internacionales que sugieren la inclusión de trabajo aeróbico y pesas en los programas de rehabilitación pulmonar, ya que mejoran la deambulación por su efecto en la fuerza y la resistencia muscular (Hindelang et al., 2020).

Se comprobó que la movilidad mejoró después de haber participado en el PRFAMEPC. Este cambio positivo se dio dada una serie de posibles modificaciones en la fuerza, la velocidad, la coordinación y el balance postural de los participantes. Mkacher et al. (2015) concluyeron que las personas adultas mayores aumentaron su equilibrio por un incremento en su fuerza muscular, como resultado de su participación en un programa de entrenamiento físico; a propósito, las mejoras en el equilibrio conllevan progreso en la 
estabilidad y movilidad, reduciendo posibles caídas, factor importante en el adulto mayor frágil (Mkacher et al., 2015; Ramírez y Coto, 2017).

De la misma manera, se ha documentado en la literatura cómo la mejora en la tolerancia al ejercicio impacta sobre la agilidad, cuando se incluyen trabajos de equilibrio en los programas de entrenamiento (Paz et al., 2014). Similarmente, en este caso, la forma como se diseñó y estructuró el programa contribuyó en la mejora de la movilidad. De manera complementaria, la aplicación de los 4 pilares del movimiento (i. e., cambios de nivel, tracción, empuje y rotación) produce que se mejore el manejo del espacio y la propiocepción, provocando que las personas vivencien un impacto positivo en su capacidad funcional, coordinación y fuerza muscular (De Lima et al., 2019).

\section{Conclusiones}

Se desprende de lo indagado que la prescripción efectiva de ejercicio para las personas adultas mayores con enfermedad pulmonar crónica incluye programar tareas aeróbicas, contrarresistencia (pesas), funcionales y fisioterapia respiratoria. Igualmente, el efecto positivo del ejercicio físico en la fuerza muscular y movilidad parece ser el resultado de un manejo adecuado de cargas de trabajo y especificidad, lo que permitió una readaptación al esfuerzo físico y, por ende, una mejora en la tolerancia a este. Sin embargo, se debe realizar más investigación sobre la efectividad de este programa, con el fin de examinar con más detalle su efecto sobre otras variables de interés; además, existen particularidades fisiológicas asociadas a los problemas restrictivos y obstructivos que deben ser revisadas durante la labor evaluativa y prescriptiva.

Es importante mencionar que los hallazgos de este estudio deben ser valorados con cuidado, pues hay algunas limitaciones metodológicas que deben ser reconocidas, incluyendo que los datos fueron recolectados con fines clínicos y no de indagación. Asimismo, se debe considerar que el tamaño muestral es reducido y se realizó un análisis conjunto de información de participantes con enfermedad pulmonar crónica.

Los aportes de este trabajo transcienden el plano investigativo, ya que los resultados podrían considerarse a la hora de diseñar programas de ejercicio enfocados en personas adultas mayores con enfermedad pulmonar crónica, porque ofrecen un insumo sobre la importancia de la combinación efectiva de tareas específicas y cargas.

\section{REFERENCIAS}

American Association of Cardiovascular \& Pulmonary Rehabilitation (AACVPR). (2011). Guidelines for pulmonary rehabilitation programs/American Association of Cardiovascular and Pulmonary Rehabilitation (AACVPR). Human Kinetics.

Caja Costarriccense de Seguro Social (CCSS). (2016). Egreso hospitalario según diagnóstico principal CCSS. Anuario estadistico. http://www.ccss.sa.cr/est_salud

De Brandt, J., Spruit, M. A., Hansen, D., Franssen, F. M. E., Derave, W., Sillen, M. J. H. y Burtin, C. (2018). Changes in lower limb muscle function and muscle mass following exercise-based interventions in patients with chronic obstructive pulmonary disease: A review of the English-language literature. Chronic Respiratory Disease, 15(2), 182-219. https://doi.org/10.1177/1479972317709642

De Lima, F. F., Camillo, C. A., Grigoletto, I., Uzeloto, J. S., Vanderlei, F. M., Ramos, D. y Cipulo, E. M. (2019). Effects of combining functional exercises with exercise training on daily physical activities and functionality in patients with COPD: a protocol for a randomized clinical trial. Trials, 20, 680. http://doi.org/10.1186/s13063-019-3 $780-\mathrm{y}$

Güell, M. R., Díaz, S., Rodríguez, G., Morante, F., San Miguel, M., Cejudo, P. Ortega, F., Muñoz, A., Galdiz, J.B., García, A. y Servera, E. (2014). Rehabilitación respiratoria. Bronconeumol., 50(8), 332-44. https://doi.org/10. 1016/j.arbres.2014.02.014 
Hindelang, M., Kirsch, F. y Leidl, R. (2020): Effectiveness of nonpharmacological COPD management on healthrelated quality of life - a systematic review. Expert Review of Pharmacoeconomics \& Outcomes Research, 20(1), 79-91. https://doi.org/10.1080/14737167.2020.1734455

Hospital Nacional de Geriatría y Gerontología (HNGG). (2016). Comisión Local de Vigilancia Epidemiológica. Costa Rica

Janaudis-Ferreira, T., Hill, K., Goldstein, R. S., Robles-Ribeiro, P., Beauchamp, M. K., Dolmage, T.E. , Wadell, K. y Brooks, D. (2011). Resistance Arm Training in Patients With COPD. A Randomized Controlled Trial. Chest, 139(1), 151 - 158. https://doi.org/10.1378/chest.10-1292

Jones, C. J., Rikli, R. E., Max, J. y Noffal, G. (1998). Reliability and validity of a chair sit- and-reach test as a measure of hamstring flexibility in older adults. Res. Q. Exerc. Sport, 69(4), 338-343. https://doi.org/10.1080/0270136 7.1998.10607708

Marco, E., Coll-Artés, R., Marín, M., Coll-Fernández, R., Pascual, M. T., Resa, J. y Círiah, M. (2016). Recomendaciones sobre programas de rehabilitación pulmonar en pacientes con enfermedad pulmonar obstructiva crónica de la Sociedad de Rehabilitación Cardiorrespiratoria. Rehabilitación, 50(4), 233-262. http: //doi.org/10.1016/j.rh.2016.04.004

McCarthy, B., Casey, D., Devane, D., Murphy, K., Murphy, E. y Lacasse, Y. (2015). Pulmonary rehabilitation for chronic obstructive pulmonary disease (Review). Cochrane Database of Systematic Reviews, 23(2). https://doi.o rg/10.1002/14651858.CD003793.pub3

Miranda, G., Gómez, A., Pleguezuelos, E. y Capellas, L. (2011). Rehabilitación respiratoria en Espana. Encuesta SORECAR. Rehabilitación, 45(3), 185-82. https://doi.org/10.1016/j.rh.2011.04.004

Mkacher, W., Mekki, M., Tabka, Z. y Trabelsi, Y. (2015). Effect of 6 Months of Balance Training During Pulmonary Rehabilitation in Patients With COPD. .. Cardiopulm. Rehabil.Prev., 35(3), 207-213. https://doi.org/10.109 7/HCR.0000000000000109.

Moore, G., Durstine, L. y Painter, P. (2016). ACSM's Exercise Management for Persons With Chronic Diseases and Disabilities. Human Kinetics.

Morales, F., Hernández, P. C. y Quesada, M. (2017). La fragilidad en la persona adulta mayor. En F. Morales. (Ed.), Tratado de Geriatria y Gerontología (pp. 269-274). EDNASSS.

Osness, W. H., Adrian, M., Hoeger, W., Rabb, D. y Wiswell, R. (1996). Functional fitness assessment for adults over 60 years: A field Based Assessment. The American Alliance for Health, Physical Education, Recreation and Dance. https://files.eric.ed.gov/fulltext/ED324293.pdf

Paz, E., López, A., González, L., Souto, S., y Fernández, R. (2014). Efectos de la rehabilitación pulmonar de corta duración en pacientes con EPOC. Fisioterapia, 37(5), 246-256. http://doi.org/10.1016/j.ft.2014.10.003

Podsiadlo, D. y Richardson, S. (1991). The timed "up and go": A test of basic functional mobility elderly persons... Am. Geriatr. Soc., 39(2),142-48. http://doi.org/10.1111/j.1532-5415.1991.tb01616.x

Rámirez, U. y Coto, F. (2017). Caídas en personas adultas mayores. En F. Morales. (Ed.), Tratado de Geriatría y Gerontologia (pp. 269-274). EDNASSS.

Rayo, D. (2013). Informe Estado de la Nación. Costa Rica: Evolución de la mortalidad y los días de estancia por egreso hospitlarios en el periodo 2013-2030. https://estadonacion.or.cr/files/biblioteca_virtual/019/rayo_d_2013.pdf

Ries, A., Bauldoff, G., Carlin, B., Casaburi, R., Emery, C., Mahle, R. D., Make, B., Rochester, C., Zuwallack, R. y Herrerias, C. (2007). Pulmonary Rehabilitation: Joint ACCP/AACVPR Evidence-Based Clinical Practice Guidelines. Chest, 131(5), 4-42. https://doi.org/10.1378/chest.06-2418

Rikli, R. E. y Jones, C. J. (2001). Senior Fitness Test Manual. Human Kinetics.

Satta, A., Migliori, G. B., Spanevello, A., Neri, M., Bottinelli, R., Canepari, M., Pellegrino, M.A., y Reggiani, C. (1997). Fiber types in skeletal muscles of chronic obstructive pulmonary disease patients related to respiratory function and exercise tolerance. Eur. Respir..., 10(2), 2853-60. https://doi.org/10.1183/09031936.97.10122853

Sivori, M., Almeida, M., Benzo, R., Boim, C., Brassesco, M., Callejas, O., Capparelli, I., Conti, E., Díaz, M., Draghi, J., Franco, J., Gando, S., Giuliano, G., Guida, R., Jolly, E., Pessolano, F., Rabinovich, R., Ratto, P., Rhodius, E. ... 
MHSALud, ISSN: 1659-097X, 18(1), ENEro-Junio, 2021, PP 1-11

Heyden López y Muñoz RoJas

Victorio, C. (2008). Nuevo Consenso Argentino de Rehabilitación Respiratoria, actualización. Medicina Buenos Aires, 68(4): 325-344. http://www.scielo.org.ar/pdf/medba/v68n4/v68n4a14.pdf

Spruit, M., Singh, S., Garvey, C., ZuWallack, R., Nici, L., Rochester, C., Hill, K., Holland, A., Lareau, S., Man, W., Pitta, F., Sewell, L., Raskin, J., Bourbeau, J., Crouch, R., Franssen, F., Casaburi, R., Vercoulen, J.H., Vogiatzis, I. ... Wouters, E. (2013). An official American Thoracic Society/European Respiratory Society Statement: key concepts and advances in pulmonary rehabilitation. Am... Respir. Crit.Care Med., 188(8), 13-64. https://doi.o $\mathrm{rg} / 10.1164 / \mathrm{rccm} .201309-1634 \mathrm{ST}$

CC BY-NC-ND 